Olatunji, B. O., \& Broman-Fulks, J. J. (2009). Latent structure of aversion: Taxometric exploration. Journal of Anxiety Disorders, 23(1): 87-92. (Jan 2009) Published by Elsevier (ISSN: 1873-7897).

doi:10.1016/j.janxdis.2008.04.002

\title{
Latent structure of aversion: Taxometric exploration
}

\author{
Bunmi O. Olatunji and Joshua J. Broman-Fulks
}

\begin{abstract}
Recent research has identified specific disorders that appear to be better characterized by the experience of aversion, rather than anxiety, which evolve from disgust-related affect. Three mathematically distinct taxometric procedures - MAXEIG, MAMBAC, and L-Mode - were applied to data from a large undiagnosed sample $(N=909)$ to determine whether aversion forms a distinct psychopathological category (i.e., an aversion taxon). Aversion was operationalized with self-report measures of the frequency of disgust experiences (Disgust Propensity), the perceived emotional impact of disgust experiences (Disgust Sensitivity), and contamination ideation and excessive washing (Contamination Fear). The collective results across procedures, consistency tests, and analysis of simulated comparison data produced converging evidence in support of the conclusion that the latent structure of aversion is dimensional. Implications of these findings for the clinical assessment of aversion and its disorders are discussed.
\end{abstract}




\section{INTRODUCTION}

Although a continuum model of anxiety has been proposed (e.g., Eysenck, 1981), a behavioral inhibition syndrome that is qualitatively distinct from other fearful behaviors has also been proposed (Kagan, 1994). These conflicting theoretical models has lead to the critical empirical question of whether pathological and normative forms of anxiety can be clearly and qualitatively discriminated. Taxometrics is a branch of applied mathematics designed specifically to discern the latent structure (i.e., categorical vs. continuous distribution) of phenomena by evaluating whether the numerical relations among various indicators of a conjectured taxon fit a pattern consistent with a naturally occurring class or a continuous dimension (Waller \& Meehl, 1998). Research addressing the question of the latent structure of anxiety and associated forms of psychopathology with taxometric procedures has the potential to inform conceptualization, investigation, assessment, and treatment of such problems, and research to date have yielded some interesting results. For example, whereas taxometric research has suggested that worry (Ruscio, Borkovec, \& Ruscio, 2001), posttraumatic stress reactions (e.g., Broman-Fulks et al., 2006), and social anxiety (Kollman, Brown, Liverant, \& Hofmann, 2006) appear to be continuous phenomena, a recent taxometric study of anxiety (as operationalized by self-report measures of subjective anxiety, anxious cognitive style, physiological arousal, and anxiety-related impairment) identified a taxon (i.e., a dichotomous latent class structure) with a prevalence of approximately 11\% (Kotov, Schmidt, Lerew, \& Joiner, 2005). Furthermore, a series of studies conducted by Bernstein and colleagues (e.g., Bernstein et al., 2007) has indicated that anxiety sensitivity (i.e., fear of anxiety symptoms and a precursor to panic disorder) may also be taxonic. Thus, some anxiety-related constructs appear to be categorical at the latent level, whereas others are dimensional.

There is growing evidence that some anxiety-related conditions may be better characterized by the experience of aversion (Olatunji \& McKay, 2007). Aversion may be defined as a common experience involving revulsion marked by thoughts of contamination, somatic symptoms of nausea, subjective symptoms of disgust, and behavioral avoidance or rejection (Olatunji \& Sawchuk, 2005). Important distinctions between anxiety and aversion have been made in the literature. Most notable is that anxiety manifests as a consequence of maladaptive regulation of fear, whereas aversion manifests as a consequence of maladaptive regulation of disgust. Fear and disgust have been differentiated along dimensions of behavioral intentions, physiological processes, and appraisal. For example, fear-based avoidance functions to protect from perceived danger, while disgust-based avoidance is often more linked to sensation or imagery (Woody \& Teachman, 2000). Additionally, the experience of fear is associated with sympathetic activation, whereas disgust is associated with parasympathetic activation (Levenson, 1992).

The distinction between fear and disgust has prompted the observation that maladaptive regulation of these two basic emotions may give rise to different disorders (Olatunji \& Sawchuk, 2005). Although it is well established that some anxiety conditions (i.e., panic disorder) are marked by an underlying maladaptive regulation of fear, there is growing evidence that other disorders (i.e., blood-injection-injury phobia; contamination-based obsessive-compulsive disorder) may be better conceptualized as aversion conditions in that they are marked by an underlying maladaptive regulation of disgust. Neuroimaging research also offers some 
supportive evidence for this distinction. Although there is ample evidence for the amygdala's role in the fear response in panic disorder (Windmann, 1998), recent evidence suggests that the defense response in contamination-based obsessive-compulsive disorder is signaled by disgust which is regulated by the insula (Husted, Shapira, \& Goodman, 2006).

Fear-based anxiety and disgust-based aversion appear to be characterized by differences in phenomenology, including physiology (sympathetic vs. parasympathetic) and underlying neural substrate (amygdala vs. insula). Recent evidence suggests that the underlying latent structure of anxiety-related variables may consist of pathological and normative forms than can be qualitatively discriminated (Bernstein et al., 2007). However, a continuous or categorical model of aversion has yet to be examined in the literature. Therefore, the goal of the present study was to evaluate the latent structure of aversion for taxonicity or dimensionality using, different selfreport indicators, multiple taxometric methods with data from a large and unscreened sample likely to evidence a wide range of aversion symptom severity.

\section{METHOD}

\subsection{Participants and procedure}

Participants consisted of 909 (58\% female) undergraduates who participated in exchange for course credit. Participants were not screened for the presence (or absence) of psychological disorders. The participants were mostly Caucasian (76\%), ranged in age from 18 to 47 (M = 19.16, S.D. $=2.11$ ), and completed the following measures in small groups:

The Disgust Propensity and Sensitivity Scale-Revised (DPSS-R; van Overveld, de Jong, Peters, Cavanagh, \& Davey, 2006) is a 16 item measure with two subscales designed to assess the frequency of disgust experiences (Disgust Propensity) and the emotional impact of disgust experiences (Disgust Sensitivity).

The Padua Inventory (PI; Burns, Keortge, Formea, \& Sternberger, 1996) contamination subscale consists of 10 items assessing contamination ideation and excessive washing concerns.

\subsection{Statistical analyses}

Three mathematically independent taxometric procedures were used to test the latent structure of aversion-MAXEIG, MAMBAC, and L-Mode. Taxometric procedures provide an estimate of the taxon base rate, if one exists. Thus, if aversion was found to be taxonic, the use of a large non-clinical (or non-diagnosed) sample would allow for an estimation of the taxon base rate in the general population. For convenience purposes, a brief explanation of the mathematical rationale behind each taxometric procedure is provided below.

MAXEIG (MAXimum EIGenvalue; Waller \& Meehl, 1998) is a multivariate procedure which calculates the eigenvalues of all remaining indicators across successive regions of an input 
indicator. The resulting graph generally takes one of two shapes. If the indicators are measuring a latent class, eigenvalues should be close to zero within each group because there should be little or no correlation between indicators within the taxon and nontaxon groups. However, eigenvalues are expected to be highest in the intervals that contain equal numbers of members from both the taxon and nontaxon groups. Therefore, the resulting graph will convex upward and peak at the interval that best discriminates the two groups. In contrast, if individuals are sampled from a single population (i.e., the construct represents a latent dimension), eigenvalues will be relatively stable across successive overlapping regions of the input indicator.

Consequently, the resulting MAXEIG graph would be relatively flat, with slight variations around a mean eigenvalue. MAXEIG was selected as the primary method of analysis, rather than the commonly used MAXCOV procedure (of which MAXEIG is a multivariate extension), because it generates a greater number of data points than MAXCOV and is more robust to indicator skew in samples where the predicted taxon base rate (if a taxon exists) would be relatively low (Ruscio \& Ruscio, 2002).

MAMBAC (Mean Above Minus Below A Cut; Meehl \& Yonce, 1994) is based on the assumption that if two discrete groups exist (i.e., taxon and nontaxon), mean differences between groups will occur on valid indicators of group membership. The MAMBAC procedure sorts all cases in ascending order on one indicator variable, computes the means of a second indicator below and above the cut, and then subtracts the mean of the second indicator below the cut from the mean above the cut. If the variable under investigation is categorical, the greatest mean differences will occur at the cut that best discriminates between the two groups, thus producing a hillshaped curve with the peak located at the cut that best differentiates the two groups. In contrast, if the variable is dimensional, the largest mean differences will occur at the extreme high and low values, resulting in a concave, or dish-shaped plot.

L-Mode (Latent-Mode Factor Analysis; Waller \& Meehl, 1998) is a multivariate taxometric procedure that performs an exploratory factor analysis on all of the candidate indicators and plots the factor score estimates for the first principal factor. When the variable under investigation is taxonic, L-Mode plots will depict a bimodal distribution of scores. In contrast, dimensional data tend to yield unimodal distributions (often centered around 0.00).

Simulated taxonic and dimensional data plots were also generated via each of the taxometric procedures using data with similar distributional characteristics (i.e., skew, kurtosis, N, etc.) to the empirical data (Ruscio, Haslam, \& Ruscio, 2006; Ruscio, Ruscio, \& Meron, 2007). Simulations can be used to ensure the appropriateness of the empirical data for taxometric evaluation (i.e., simulated taxonic and dimensional plots should be clearly distinguishable) and aid in the interpretation of the empirical data plots (i.e., provide comparison plots for the experimental data plots). Cases were sorted into putative taxon and nontaxon membership using Bayes' Theorem in the MAXEIG program, the grand mean base rate estimate in MAMBAC, and the nearest mode in L-Mode. These programs also provide a Comparison Curve Fit Index (CCFI), which is an objective numerical gauge of whether the data plots are more consistent with a taxon or dimension. The CCFI scores range between 0 and 1 , with higher scores (i.e., >0.5) being suggestive of a taxon, and lower scores (<0.5) representing support for 
a dimensional solution. Empirical support for the validity of the CCFI has been provided in recent simulation studies (e.g., Ruscio et al., 2007).

\subsection{Indicator selection}

Three indicators were generated by summing the items representing the two DPSS-R subscales and the PI contamination fear subscale. These indicators were chosen as they converge on unique aspects related to the experience of aversion. Aversion, or an intense sense of repugnance, may be marked by frequent experiences of repulsion (Disgust Propensity), a perceived negative impact of experiencing repulsion (Disgust Sensitivity), and contamination concerns (Contamination Fear). As shown in Table 1, the three indicators demonstrated good internal consistency and were significantly correlated with one another. The three indicators also demonstrated low nuisance correlations (taxon mean $=.23$ and nontaxon mean $=.20$ ), high validity $(M=1.84)$, and moderate to low skew $(M=.63)$, suggesting that they were suitable for taxometric analysis. A suitability analysis, which involves examining the simulated taxonic and dimensional plots produced by each taxometric procedure to ensure that the data is capable of distinguishing between a taxon and dimension, confirmed the appropriateness of the research data for taxometric analysis.[1]

Table 1. Descriptive data, psychometric characteristics and correlation coefficients of the three aversion indicators

\begin{tabular}{|c|c|c|c|c|c|c|c|c|c|c|}
\hline Indicator & Mean & S.D. & $\alpha$ & Range & $\begin{array}{l}\text { Indicator } \\
\text { validity }\end{array}$ & Skew & Kurtosis & 1 & 2 & 3 \\
\hline $\begin{array}{l}\text { 1. Disgust } \\
\text { Propensity }\end{array}$ & 15.52 & 6.60 & 0.90 & $\begin{array}{l}0.00- \\
40.00\end{array}$ & 2.04 & 0.36 & 0.34 & - & $0.83^{*}$ & $0.40^{*}$ \\
\hline $\begin{array}{l}\text { 2. Disgust } \\
\text { Sensitivity }\end{array}$ & 12.10 & 6.78 & 0.88 & $\begin{array}{l}0.00- \\
38.00\end{array}$ & 2.12 & 0.42 & 0.06 & & - & $0.40^{*}$ \\
\hline $\begin{array}{l}\text { 3. Contamination } \\
\text { Fear }\end{array}$ & 9.20 & 5.47 & 0.90 & $\begin{array}{l}0.00- \\
37.00\end{array}$ & 1.38 & 1.12 & 0.83 & & & - \\
\hline
\end{tabular}

Note: ${ }^{*} p<.001$

\section{RESULTS}

MAXEIG was applied to the three indicators. None of the resulting plots demonstrated evidence of taxonicity as evidenced by the lack of any clear peaks (see Fig. 1).[2] Rather, all three plots were relatively flat with minor fluctuations. Furthermore, the empirical data plots, including an 
averaged curve, closely resembled the simulated dimensional plots and were inconsistent with the clear peaks noted in the simulated taxonic plots. The CCFI for the aversion data was 0.21 , which supports the existence of a latent dimension. Thus, results of the MAXEIG analyses provided initial evidence for a dimensional conceptualization of aversion.
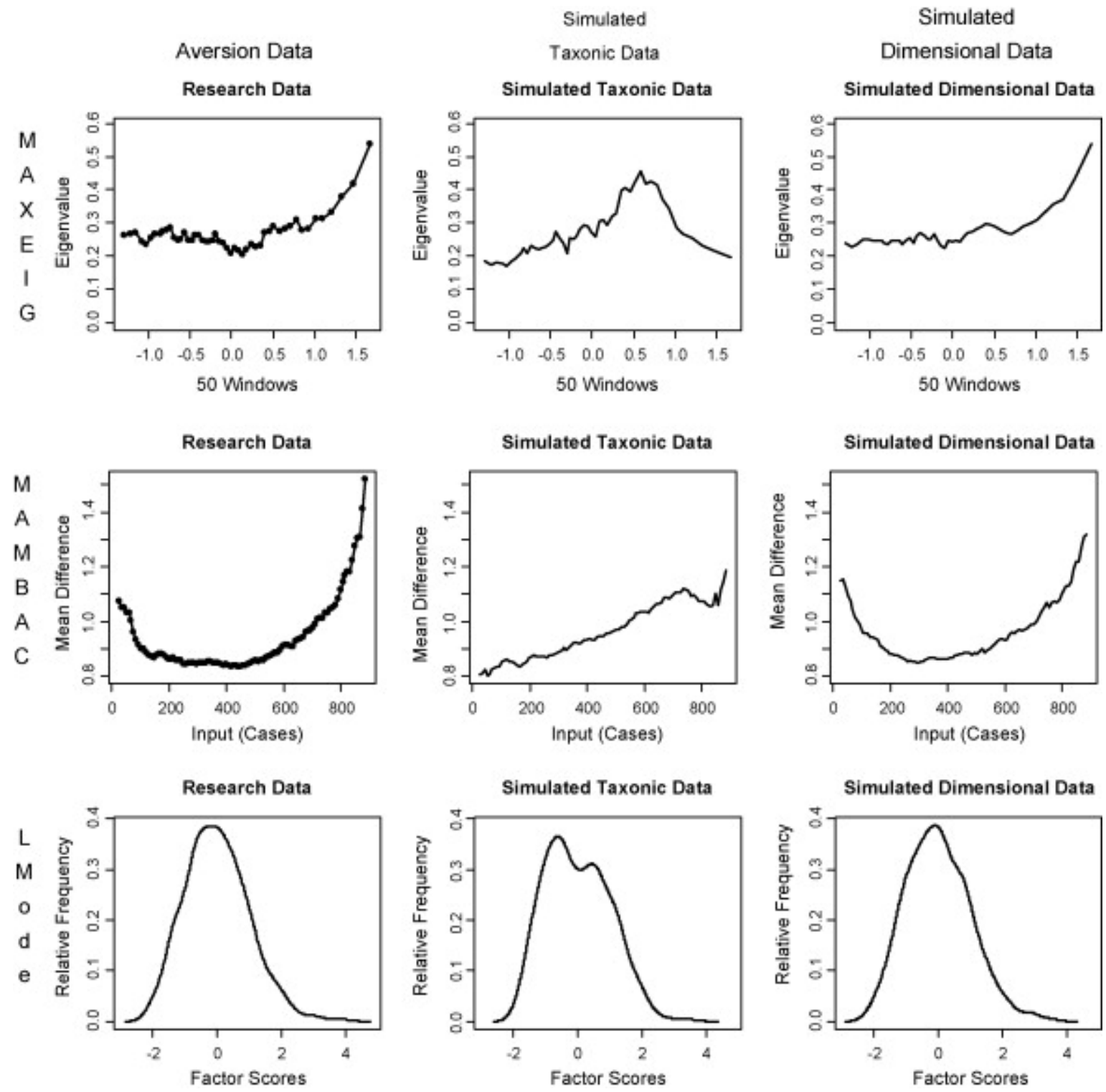

Fig. 1.

Averaged MAXEIG (top), MAMBAC (middle), and L-Mode (bottom) plots for the aversion data (left), simulated taxonic data (center), and simulated dimensional data (right). 
Taxometric procedures rely heavily on the replication of findings across independent procedures to ensure accuracy of results. Thus, MAMBAC and L-Mode analyses were also conducted on the aversion data as additional consistency tests. MAMBAC analyses generated six plots, all of which were relatively flat or dish shaped, and closely matched the simulated dimensional data plots. The CCFI for the MAMBAC analyses was 0.30. Similarly, L-Mode analysis of the empirical data revealed a unimodal distribution, which can be contrasted with the typical bimodal distribution of taxonic data and the simulated taxonic plot. Thus, results of MAMBAC and LMode analyses were consistent with the MAXEIG findings and provided convergent evidence for a dimensional conceptualization of aversion.

In addition to producing data plots, each taxometric procedure also provides one or more estimates the base rate of the proposed taxon (if one exists). Inspection of the three MAXEIG base rate estimates indicated considerable variability, ranging from 0.09 to $0.49(M=0.27$, S.D. $=.20)$. The six MAMBAC base rate estimates were more consistent $(M=0.42$, S.D. $=0.07)$ though substantially higher than the theoretically expected rate of aversion in a non-clinical sample. Finally, the L-Mode analysis indicated a base rate of 0.51 , which is also consistent with latent dimensional variables.

\section{DISCUSSION}

Recent research suggests that the latent structure of anxiety is taxonic, such that a non-arbitrary latent category whose members are qualitatively distinct from nonmembers can be identified (Kotov et al., 2005). In addition, a recent study in a large non-clinical sample found a latent fear of anxiety symptoms taxon that demonstrated incremental validity in predicting future panic attacks (Schmidt, Kotov, Lerew, Joiner, \& Ialongo, 2006). The fear of anxiety symptoms taxon also appears to function as a diathesis for posttraumatic stress disorder (PTSD; Bernstein et al., 2005). These findings highlight the distinctiveness of anxiety and disorders that are based on maladaptive regulation of fear. However, recent developments suggest that some disorders may reflect an aversion mood state based on maladaptive regulation of disgust (Olatunji \& McKay, 2007). Although distinctions have been made between fear-based anxiety and disgust-based aversion with regards to subjective, cognitive, physiological, and neurobiological correlates (Olatunji \& Sawchuk, 2005), it is unclear if anxiety and aversion can also be distinguished based on differences in latent structure.

The present investigation uniquely addressed absence of empirical evidence that bears directly on the latent continuity of aversion by conducting a taxometric analysis in a large non-clinical sample. Specifically, the latent structure of aversion was examined by applying three taxometric procedures - MAXEIG, MAMBAC, and L-Mode - to a set of aversion indicators consisting of the frequency of disgust experiences, the perceived emotional impact of disgust experiences, and contamination ideation and excessive washing. None of the taxometric procedures produced evidence for a latent aversion taxon. Rather, the results were consistent with a dimensional solution for aversion, as indicated by characteristically dimensional plot shapes and base rate estimates that were highly discrepant from the prevalence of aversion in the respective populations. 
If taxonicity reflects the true latent structure of the experience of anxiety (Kotov et al., 2005), then these findings of dimensionality serve to further highlight the distinctiveness of aversion from anxiety. The distinction between anxiety and aversion in terms of latent structure may largely derive from differences in the underlying basic emotions from which the two negative mood states originate. While anxiety originates from fear, aversion appears to originate from disgust (Olatunji \& Sawchuk, 2005). Although fear and disgust are both negative emotions, important distinctions have been made between the two emotions across several dimensions (Woody \& Teachman, 2000). When considered in the context of the available literature suggesting taxonicity for the structure of anxiety, the present findings suggest that perhaps the pathological consequences of maladaptive regulation of fear can also be distinguished from that of the maladaptive regulation of disgust by the assessment of underlying latent structure.

Presence of an anxiety taxon documented in the literature and the absence of an aversion taxon found in the present study has important implications for the clinical assessment of related conditions. To the extent that anxiety is taxonic, systematic screening for individuals that fall in an anxiety taxon could facilitate more efficient (i.e., sensitivity and specificity) identification of individuals at risk for the development of fear-related conditions such as panic disorder (Schmidt et al., 2006) or PTSD (Bernstein et al., 2005). However, various conditions (i.e., spider phobia, blood-injection-injury phobia, contamination-based obsessive-compulsive disorder, eating disorders, hypochondriasis, body dysphormic disorder, sexual aversion disorder, emetophobia, etc.) are considered disorders of aversion in that they are largely mediated by disgust (Phillips, Senior, Fahy, \& David, 1998). Consistent with the present findings, recent research examining the latent structure of some aversion-based disorders have also found supportive evidence for dimensionality (i.e., Haslam, Williams, Kyrios, McKay, \& Taylor, 2005). The dimensional solution for aversion and related conditions indicates that the use of continuous measures focused on evaluating the full range of symptoms is appropriate and will maximize statistical power and minimize information loss. Future research may then assess if consideration of the full range of aversion facilitates the application of current theoretical approaches to account for aversion indicators (i.e., Disgust Sensitivity) across a broader range of disorders in diverse populations.

These findings also point to the possibility that incorporation of information regarding the apparent difference in latent structure of fear-based anxiety and related conditions and disgustbased aversion and associated conditions into clinical assessment may potentially render intervention programs more effective. However, these results should be interpreted within the context of the study limitations. The sample reflected a very specific non-clinical participant population (undergraduate students). Consequently, the generalizability of our findings is very limited. However, it should be noted that approximately $14 \%$ of our sample scored above the OCD washer patient mean reported on the PI contamination subscale (mean $=13.87$; Burns et al., 1996). Therefore, it appears unlikely that the current results were due to the exclusive reliance on non-clinical data. However, the indicators used in this study were limited to selfreport instruments that define a narrow aversion construct. Future research incorporating different measures, different measurement modalities (i.e., physiological parameters of nausea), and a broader set of taxometric and nontaxometric procedures will be vital in making more definitive inferences regarding the latent structure of aversion as differentiated from anxiety, as 
well as the basic emotions from which they originate. This line of research may ultimately have implications for the refinement of the existing taxonomy of affective disorders.

\section{NOTES}

1. Three experienced taxometricians, including two external consultants, examined the simulations and confirmed that the simulated taxonic and dimensional plots clearly distinguishable.

2. The three taxometricians also rated the aversion data plots for evidence of taxonicity, dimensionality, or ambiguity. Results indicated that the raters were in complete agreement that the aversion data plots were suggestive of a latent dimension.

\section{ACKNOWLEDGEMENTS}

We thank Bradley A. Green and Randolph C. Arnau for their assistance with plot ratings.

\section{REFERENCES}

Bernstein et al., 2005 A. Bernstein, M.J. Zvolensky, M.T. Feldner, S.F. Lewis, A.L. Fauber and E.W. Leen-Feldner, et al. Anxiety sensitivity taxon and trauma: Discriminant associations for posttraumatic stress and panic symptomatology among young adults. Depression and Anxiety, 22 (2005), pp. 138-149.

Bernstein et al., 2007 A. Bernstein, M.J. Zvolensky, P.J. Norton, N.B. Schmidt, S. Taylor and J.P. Forsyth, et al. Taxometric and factor analytic models of anxiety sensitivity: Integrating approaches to latent structural research. Psychological Assessment, 19 (2007), pp. 74-87.

Broman-Fulks et al., 2006 J.J. Broman-Fulks, K.J. Ruggiero, B.A. Green, D.G. Kilpatrick, C.K. Danielson and H.S. Resnick, et al. Taxometric investigation of PTSD: Data from two nationally representative samples. Behavior Therapy, 37 (2006), pp. 364-380.

Burns et al., 1996 G.L. Burns, S.G. Keortge, G.M. Formea and L.G. Sternberger, Revision of the Padua inventory of obsessive compulsive disorder symptoms: Distinctiveness between worry, obsessions, and compulsions. Behaviour Research and Therapy, 34 (1996), pp. 163-173.

Eysenck, 1981 H.J. Eysenck, A model of personality, Springer-Verlag, New York (1981).

Haslam et al., 2005 N. Haslam, B.J. Williams, M. Kyrios, D. McKay and S. Taylor, Subtyping obsessive-compulsive disorder: A taxometric analysis. Behavior Therapy, 36 (2005), pp. 381391. 
Husted et al., 2006 D. Husted, N. Shapira and W. Goodman, The neurocircuitry of obsessivecompulsive disorder and disgust. Progress in Neuro-Psychopharmacology and Biological Psychiatry, 30 (2006), pp. 389-399.

Kagan, 1994 J. Kagan, Galen's prophecy, Basic Books, New York (1994).

Kollman et al., 2006 D.M. Kollman, T.A. Brown, G.I. Liverant and S.G. Hofmann, A taxometric investigation of the latent structure of social anxiety disorder in outpatients with anxiety and mood disorders. Depression and Anxiety, 23 (2006), pp. 190-199.

Kotov et al., 2005 R. Kotov, N.B. Schmidt, D.R. Lerew and T.E. Joiner, Latent structure of anxiety: Taxometric exploration. Psychological Assessment, 17 (2005), pp. 369-374.

Levenson, 1992 R.W. Levenson, Autonomic nervous system differences among emotions. Psychological Science, 3 (1992), pp. 23-28.

Meehl and Yonce, 1994 P.E. Meehl and L.J. Yonce, Taxometric analysis I: Detecting taxonicity with two quantitative indicators using means above and below a sliding cut (MAMBAC procedure). Psychological Reports, 74 (1994), pp. 1059-1274.

Olatunji and McKay, 2007 B.O. Olatunji and D. McKay, Disgust and psychiatric illness: Have we remembered?. British Journal of Psychiatry, 190 (2007), pp. 457-459.

Olatunji and Sawchuk, 2005 B.O. Olatunji and C.N. Sawchuk, Disgust: Characteristic features, social implications, and clinical manifestations. Journal of Social and Clinical Psychology, 24 (2005), pp. 932-962.

Phillips et al., 1998 M.L. Phillips, C. Senior, T. Fahy and A.S. David, Disgust-The forgotten emotion of psychiatry. British Journal of Psychiatry, 173 (1998), pp. 373-375.

Ruscio et al., 2001 A.M. Ruscio, T.D. Borkovec and J. Ruscio, A taxometric investigation of the latent structure of worry. Journal of Abnormal Psychology, 110 (2001), pp. 414-422.

Ruscio et al., 2006 J. Ruscio, N. Haslam and A.M. Ruscio, Introduction to the taxometric method: A practical guide, Lawrence Erlbaum Associates, Mahwah, NJ (2006).

Ruscio and Ruscio, 2002 A.M. Ruscio and J. Ruscio, The latent structure of analogue depression: Should the Beck Depression Inventory be used to classify groups?. Psychological Assessment, 14 (2002), pp. 135-145.

Ruscio et al., 2007 J. Ruscio, A.M. Ruscio and M. Meron, Applying the bootstrap to taxometric analysis: Generating empirical sampling distributions to help interpret results. Multivariate Behavioral Research, 42 (2007), pp. 349-386.

Schmidt et al., 2006 N.B. Schmidt, R. Kotov, D.R. Lerew, T.E. Joiner and N.S. Ialongo, Evaluating latent discontinuity in cognitive vulnerability to panic: A taxometric investigation. Cognitive Therapy and Research, 29 (2006), pp. 673-690. 
van Overveld et al., 2006 M. van Overveld, P.J. de Jong, M.L. Peters, K. Cavanagh and G.C.L. Davey, Disgust propensity and disgust sensitivity: Separate constructs that are differentially related to specific fears. Personality and Individual Differences, 41 (2006), pp. 1241-1252.

Waller and Meehl, 1998 N.G. Waller and P.E. Meehl, Multivariate taxometric procedures: Distinguishing types from continua, Sage, Newberry Park, CA (1998).

Windmann, $1998 \mathrm{~S}$. Windmann, Panic disorder from a perspective integrating neurobiological and psychological approaches. Journal of Anxiety Disorders, 12 (1998), pp. 485-507.

Woody and Teachman, 2000 S.R. Woody and B.A. Teachman, Intersection of disgust and fear: Normative and pathological views. Clinical Psychology: Science and Practice, 7 (2000), pp. 291-311. 